\title{
Nonlinear Sagnac filter in eight figure multi- wavelength fiber laser based EDF
}

\begin{abstract}
Eight figure fiber Laser design employed for generation of the multi-wavelength laser with so stable narrow line widths. In this research, the setup of multi-wavelength fiber laser source is demonstrated as well as eight figure cavity by using a piece of Polarization Maintaining Fiber (PMF) in Sagnac filter that achieved four stable and constant amplitude wavelengths with same spacing at room temperature. Also, we proposed and compared more than 250 narrow line width modes by using the $23 \mathrm{~km}$ length of Single Mode Fiber (SMF-28) at C and L communication windows. In this case, the stability of modes due to increasing the length was less in compare to PMF Sagnac.
\end{abstract}

Keywords: eight figure, polarization maintaining fiber, Sagnac filter, multi-wavelength fiber laser

\author{
Volume I Issue 3 - 2017
}

\section{HR Zare, S Shahi}

Dental Biophotonics and Laser Research Center (DBLRS),

Islamic Azad University, Iran

\begin{abstract}
Correspondence: Sharifeh Shahi, Dental Biophotonics and Laser Research Center (DBLRS), Khorasgan (Isfahan) Branch, Islamic Azad University, Arghavanieh, Isfahan, Iran,
\end{abstract} Email shahilaser@khuisf.ac.ir

Received:September 23, 2017 | Published: October 03, 2017
Abbreviations: WDM, wavelength division multiplexing systems; EDFRL, erbium doped fiber ring lasers; PC, polarization controller; PMF, Polarization maintaining fiber; OSA, optical spectrum analyzer; SNR, signal to noise ratio; SMF, single mode fiber

\section{Introduction}

Fiber lasers were made possible in the 1960s by the incorporation of trivalent rare-earth ions such as neodymium, erbium, and thulium into glass hosts. ${ }^{1}$ Starting in 1989, the focus tuned to the development of erbium doped fiber lasers since were useful for optical communications, ultrafast phenomena and fiber sensors. ${ }^{2}$ Another attractive fiber laser sources is multi-wavelength lasers that can replace Fabry-Perot lasers in Wavelength Division Multiplexing (WDM) systems. ${ }^{3}$ Ring Fiber Lasers are suitable replacement for producing the multi-wavelength laser with narrow bandwidth and mode locked laser by employing the nonlinear ring mirror filters. Erbium doped Fiber Ring lasers (EDFRLs) are highly regarded for optical communication applications because of performance at spectral range of $1550 \mathrm{~nm}$ and simultaneous operation in the range of low-loss silicon fibers. EDFRLs pumped at 980 or $1480 \mathrm{~nm}$ wavelengths because of absence of excited state absorption in the wavelengths. The selecting two pump wavelengths are always in terms of conditions, because each one has its advantages. ${ }^{4}$ The characteristics of these lasers include: very efficient operation, producing the stable wavelength, very narrow line width, high output power and tuning over a range up to $40 \mathrm{~nm} .{ }^{5} \mathrm{In}$ an ideal case, a multi-wavelength source should exhibit the following properties: $^{4}$
a. Stable power and wavelength operation.
b. Tunable over a broad wavelength range.
c. Large or small tunable wavelength spacing.
d. High output power.
e. Single mode operation.

Not all of the above features need to be satisfied simultaneously and rather it depends on the applications. Multi-Wavelength fiber laser sources ${ }^{6,7}$ vastly due to the cheapness and ease of construction are taken into consideration. These types of lasers have high potential and can be replaced by diode lasers due to their diverse applications in optical fiber sensors, spectroscopy and WDM systems. ${ }^{6}$

There are several ways to get the performance of multi-wavelength operation. One common way is to divide the broadband source spectrum for selecting the optimal wavelengths. ${ }^{4}$ In this case, various configurations like eight figures are the most common solution that adds fiber Sagnac to laser ring cavity. ${ }^{8}$ If Sagnac loop with birefringence properties added to fiber laser, coupling light between the slow and fast axes of birefringence fiber provide spectral filter. ${ }^{9}$ In Sagnac, input signal is split into two beams counter-propagation along the loop arm that after propagation in loop recombined at coupler. This interference is depending on birefringence properties of cavity and reflectivity on this mirror filter is also depending on wavelength.

In particular cavity for generation multi-wavelength laser modes employed Sagnac loop including the Polarization Maintaining Fiber as a comb filter due to its intrinsic advantages such as easy fabrication, stability and flexibility. In this case, the important points are change the length of PMF and operation temperature of comb filter .The conventional PMF loop mirror including one Polarization Controller (PC) can operate as a comb filter. The input light at coupler 50:50 is split into two beams counter-propagating in loop and the polarization states of the two beams are altered by PC. Two beams that traverse the cascaded PMF and PC in opposite directions experience different phase delays that is proportional to the product of the PMF length and the effective birefringence interference as recombined at the coupler. ${ }^{10}$ By adjusting the state of the polarization controller (PC) can be changed the state of polarization and number of lasing wavelengths. ${ }^{11}$

As shown in Figure 1, the incident wave at the coupler is split into a clockwise direction wave and an anti-clockwise one. These two counter-propagating waves undergo the same length of PMF and mode spacing of PMF follows: 


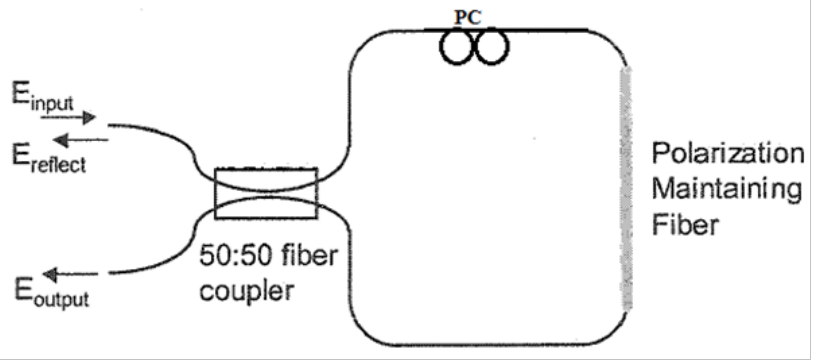

Figure I The schematic diagram of Sagnac filter.

$$
\Delta \ddot{\mathrm{e}}=\frac{\ddot{\mathrm{e}}^{2}}{2\left|\mathrm{n}_{\mathrm{s}}-\mathrm{n}_{\mathrm{f}}\right| \mathrm{L}_{\mathrm{HiBi}}}
$$

Where is the oscillating wavelength, $\mathrm{n}_{\mathrm{s}, \mathrm{f}}$ the refractive index of the slow and fast birefringence axes of the PM fiber, respectively and $\mathrm{L}_{\mathrm{HiBi}}$ is the PM fiber length.

As can be seen from the above equation, with increasing the length of PM fiber, the space of wavelengths decreased and result at constant time interval increase (increased) by the number of wavelengths. For example, two times of PMF's length with the same birefringence index in loop achieved half the mode spacing. ${ }^{11}$ In 2008, liu et al. ${ }^{11}$ demonstrated a multi-wavelength erbium-doped fiber ring laser using a fiber Sagnac loop filter. The fiber Sagnac loop filter served as the wavelength selection component within the ring laser cavity. By tuning the setting of the polarization controller (PC) to effectively change the state of polarization of the light, three, four and five lasing modes were obtained from the output port of the ring laser. ${ }^{12}$

\section{Experimental results}

The experimental setup of the proposed multi-wavelength fiber ring laser is shown in Figure 2 that is consist of 5.6 meter of Erbium fiber as a gain medium, a 10:90 coupler, a 980/1550WDM coupler as coupling pump power into the cavity, Isolator and Sagnac filter. Gain medium is placed after WDM coupler and before output coupler. Output coupler was employed to tap the 10 percent of lasing output for monitoring with an optical spectrum analyzer (OSA) and coupled the 90 percent of lasing into the cavity. Isolator can guide the light only one direction and block feedback from the WDM coupler. The Sagnac loop is formed by 3.8 meter of PMF (Panda) with birefringence index around 0.00036 that connected to the ring with 50:50 couplers. Linear polarization coupler was also used to tuning the state of polarization.

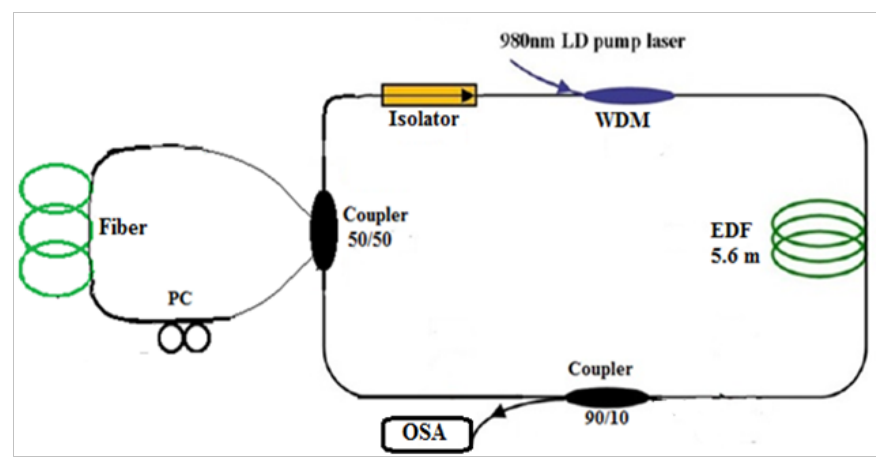

Figure 2 Schematic diagram of proposed multi-wavelength fiber ring laser.
The ASE spectrum of EDF source ${ }^{13}$ in $\mathrm{C}$ band region before completing the eight figure arrangement shown in (Figure 3). As shown in Figure 4 at low power pump (about $30 \mathrm{~mW}$ ), the filtering effect is dominated and with proper adjustment of the tension and angle of polarization controller at range $1555-1580 \mathrm{~nm}$ (C\&L bands) appeared about 10 continuous modes with same mode spacing $1.6 \mathrm{~nm}$. With increasing the power to $300 \mathrm{~nm}$ gain medium effect is dominant and energy peak powers observed at 4 excited main modes with $42 \mathrm{~dB}$ Signal to Noise Ratio (SNR) less than 3dB fluctuation at 1563.6, 1565.2, 1566.8 and $1568.5 \mathrm{~nm}$ wavelengths (Figure 5). By inserting another polarization controller (PC) to Sagnac loop mirror, more modes appeared at output that results of simulation and experimental efforts shown (Figures 6A) \& (Figure 6B), respectively.

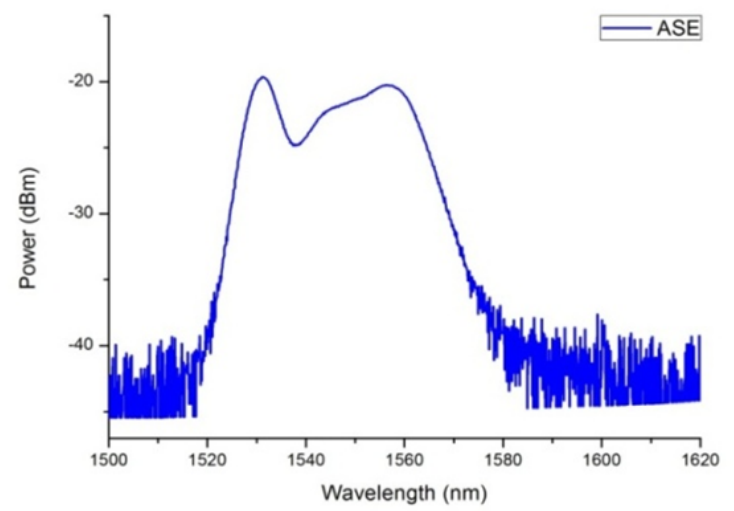

Figure 3 ASE spectrum of EDF source. ${ }^{13}$

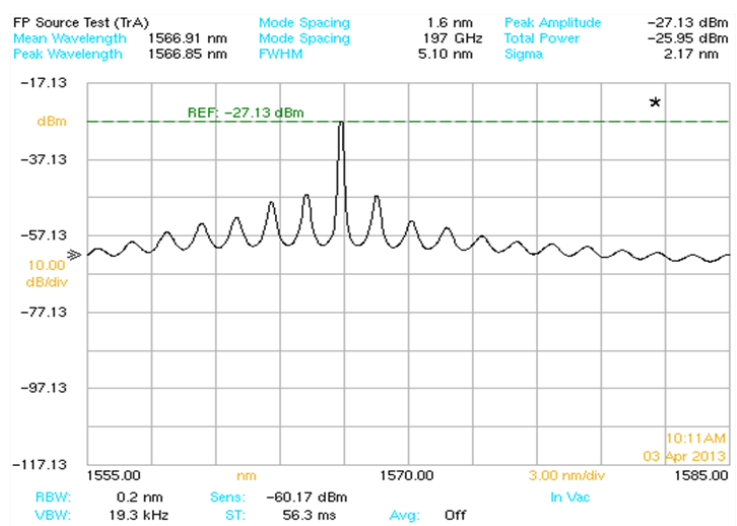

Figure 4 The output spectra of multi-wavelength fiber ring laser at pump power of $30 \mathrm{~mW}$.

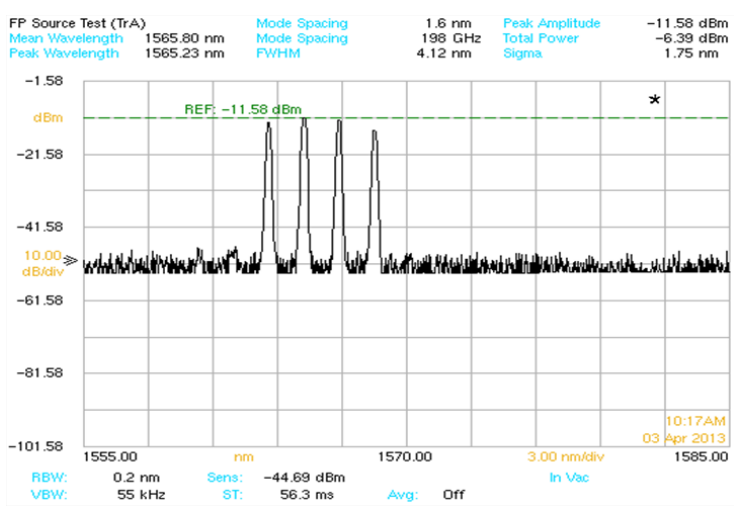

Figure 5 The output power of multi-wavelength fiber ring laser at pump power of $300 \mathrm{~mW}$. 


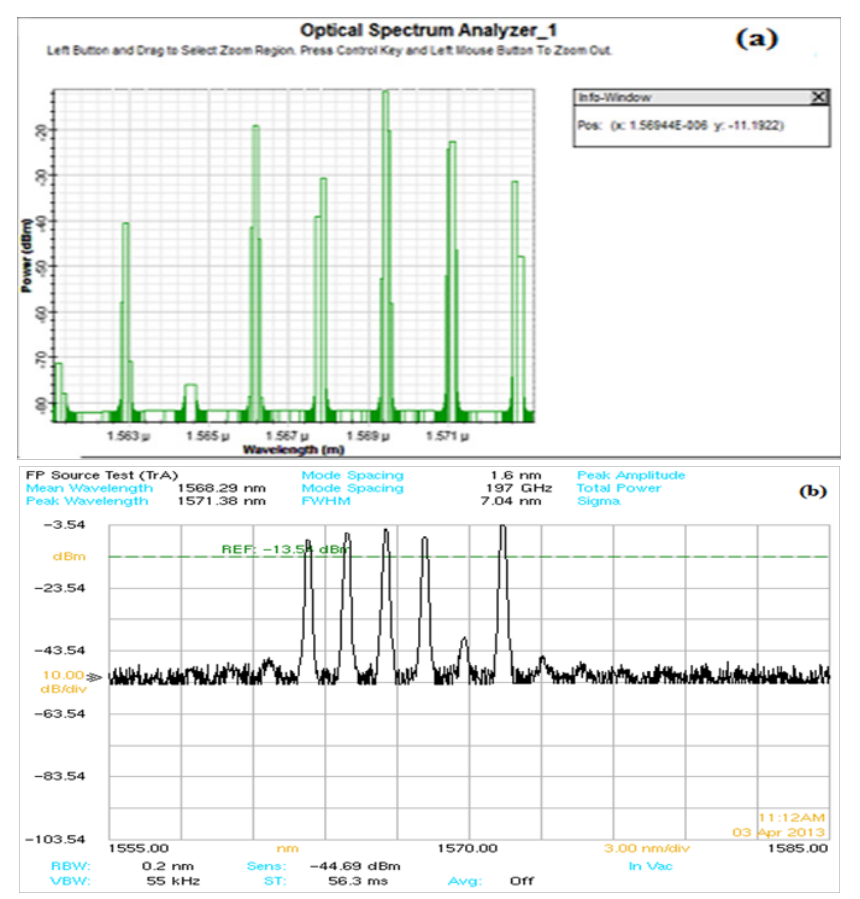

Figure 6 The spectral output power of OSA for PMF optical filter with 3.8 meter length a) Simulation b) experimental results.

Before the advent of special fibers with nonlinear, birefringence characteristic and unusual structures, the simple single mode fibers used instead of certain short fibers to improve the performance of loss, length, nonlinearity fiber and compression of lasers. In this research,

\section{Results}

In this research, the performance of continuous eight figure multi-wavelength erbium doped fiber laser demonstrated at $\mathrm{C}$ and $\mathrm{L}$ bands of telecommunication windows. The four stable and same amplitude wave with fluctuation less than $3 \mathrm{~dB}, 42 \mathrm{~dB}$ Signal to Noise Ratio (SNR) and $1.6 \mathrm{~nm}$ mode spacing achieved by employing 3.8 meter of polarization maintaining fiber in Sagnac filter. Hence, by using the birefringence PMF as a part of nonlinear loop mirror, the simple multi-wavelength filter obtained by changing tension and angle of polarization controller that the number of lasing wavelengths and mode spacing controlled. The reason of using Sagnac loop as a the $23 \mathrm{~km}$ of single mode fiber (SMF 28) also employed in Sagnac loop mirror instead of PMF and the behavior of two kinds of fibers compared. Figure 7 is shown the output spectra of $23 \mathrm{~km}$ single mode fiber. In this setup, the number of peaks increased and reached to 70 modes at wavelength interval of $12 \mathrm{~nm}$ with $0.16 \mathrm{~nm}$ mode spacing that covered $\mathrm{C}$ and $\mathrm{L}$ bands. As can be seen in Figure 7, the first stimulated Stocks appeared at $63 \mathrm{~mW}$ peak power with satisfying SMSR more than $35 \mathrm{~dB}$. In the long Single mode fibers because of cavity length loss polarization states not maintained, hence, these cases with short birefringence fibers as PMF is compensable. So, Polarization maintaining fibers as type panda are well candidate to achieve stable mode birefringence with very low losses and low crosstalk effect that have been developed in optical telecommunication and spectroscopy applications.

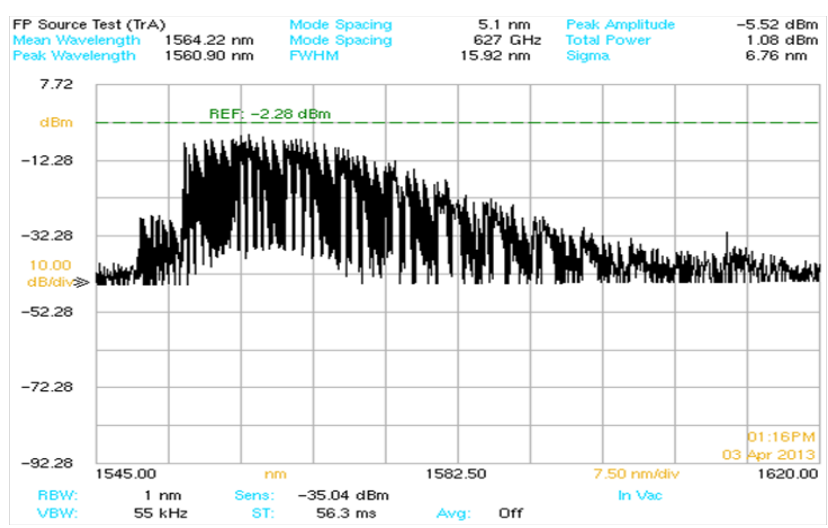

Figure 7 The output spectra of fiber ring laser and Sagnac loop consist of $23 \mathrm{~km}$ of SMF.

multi-wavelength filter is that operate independent of polarization and is equivalent to active modulators at mode locked fiber lasers. Here, the ring array as well as eight figures is simple method and low cost to operating multi wavelength fiber laser. With replacing $23 \mathrm{~km}$ of Single mode fiber (SMF-28) in Sagnac loop instead of PMF can cover $\mathrm{C}$ and $\mathrm{L}$ bands that increase the number of modes, but unstable output spectrum obtained. Table 1 compares the results of using two types fiber with different length. Design of multi-wavelength fiber ring laser has unique benefits, like simple topology, adjustable wavelength spacing and tenability lasing, small bulk and low cost in Telecommunication, Biophotonics and so on.

Table I: Comparison of performance two kinds of fiber with different lengths (PMF and SMF)

\begin{tabular}{|c|c|c|c|c|c|c|c|}
\hline $\begin{array}{l}\text { Type of Fiber in Sagnac } \\
\text { Loop }\end{array}$ & Length & $\begin{array}{l}\text { Mode } \\
\text { Spacing } \\
(\mathrm{Nm})\end{array}$ & $\begin{array}{l}\text { Number of } \\
\text { Modes }\end{array}$ & $\begin{array}{l}\text { Fluctuation } \\
\text { of Modes } \\
\text { (Db) }\end{array}$ & $\begin{array}{l}\text { OSNR } \\
\text { (Db) }\end{array}$ & $\begin{array}{l}\text { Bandwidth } \\
(\mathbf{N m})\end{array}$ & $\begin{array}{l}\text { Telecommunication } \\
\text { Window }\end{array}$ \\
\hline PMF & $3.8 \mathrm{M}$ & 1.6 & 13 & $\sim 3$ & 42 & 0.46 & $\mathrm{~L}$ \\
\hline SMF & $23 \mathrm{Km}$ & 0.16 & $\sim 70 \mathrm{ln} I 2 \mathrm{Nm}$ & $\sim 2$ & 40 & 0.14 & $C+L$ \\
\hline
\end{tabular}

\section{Acknowledgments}

None.

\section{Conflicts of interest}

The author declares there is no conflict of interest.

\section{References}

1. LE Nelson, DJ Jones, K Tamura, et al. Ultrashort-Pulse Fiber Ring Lasers. Applied Physics B. 1997;65(2):277-294.

2. GP Agrawal. Applications of Nonlinear Fiber Optics. 1st ed. Elsevier, India. 2006.

3. FW Tung. Multiwavelength Fiber Ring Lasers. The Hong Kong 
Polytechnic University, Department of Electrical Engineering, Hong Kong. 2002

4. A Hayder. Multiwavelength Brillouin Semiconductor Fiber Lasers. Department of Electrical and Computer Engineering, McGill University, Montreal, Quebec, Canada. 2008.

5. HJR Dutton. Understanding Optical Communications. 1st ed. IBM International Technical Support Organization, South Korea. 1998.

6. J Vasseur. Multiwavelength Laser Sources for Broadband Optical access Networks. Department of Electrical and Engineering of Georgia Institute of Technology, Georgia. 2006. p. 1-165.

7. K Zhou, W Ye, J Yang, et al. Comb Filter Based on an All Polarization Maintaining Fiber Loop Mirror and its Application. Application of Photonic Technology, Georgia. 2002. p.1-8.

8. MJF Digonnet. Rare-Earth Doped Fiber Lasers and Amplifiers. Marcel Dekker, Inc, New York, USA. 2001.
9. G Sun, DS Moon, A Lin, et al. Tunable Multiwavelength Fiber Laser Using a Comb Filter Based on Erbium-Ytterbium Co-doped Polarization Maintaining Fiber Loop Mirror. Optics Express. 2008;16(6):3652-3658.

10. DS Moon, U Peak, Y Chung. Polarization Controlled multi-wavelength Er-doped fiber laser using fiber Bragg grating written in few-mode sidehole fiber with an elliptical core. Optics Express. 2005;13(14):5574-5557.

11. C kim. Tunable Multiwavelength fiber lasers Based on novel Fiber-Optic Components. Section 3, UMI Baltimore, Maryland, USA. 2004.

12. Y Liu, D Liu, H Wang. Multiwavelength Erbium-Doped Fiber Ring Laser Based on a Fiber Sagnac Loop Filter. Microwave and Optical Technology Letters. 2008;50(12):3053-3054.

13. MK Jazi, S Shahi, MJ Hekmat, et al. Baghi The evaluation of various designs upon C\&L band super fluorescent sources based Erbium Doped Fiber. Laser Physics. 2013;23(6). 